\title{
Axolotl/Bichos Raros Crónica
}

\section{Susana Chávez-Silverman, Pomona College}

\author{
Buenos Aires/Los Angeles \\ 29 julio, 2001/25 mayo, 2010 \\ Para Julio Cortázar y Alejandra Pizarnik, in memoriam \\ And for Wim Lindeque and James Zike, for (y)our way of seeing
}

Viéndome sentada allí, en ese vinyl-topped, uncannily casi '50s Califas-style table, gazing embrujada into the little tank,-_Qué son? me pregunta una casi-hip, slightly concheta mujer. Me lo pregunta a mí, cual si yo fuese la dueña del lugar, de este PoMo lite, matte oxblood-red painted bar en el 'pop hotel' [sic] Boquitas Pintadas, owned by una romántica pareja de young Germans y del cual había estado leyendo todo mi año en Buenos Aires pero I'd never actually made it here, y ahora. Now, just days before leaving quiero engushirlo, engushirte Buenos Aires. Toda.

Anygüey, esta mujer asks me, casi como si yo fuese la dueña, también, de ellos. De los axolotl.

Son axolotls, le digo. Ajolotes, les dicen en México. Como en el cuento de Cortázar, ¿te acordás? Son aztecas. La mujer smiles distractedly, pero she's already backing away from me, slowly, cual si fuese sho la eccéntrica, backing up back to her comfortable table para comentar a su boyfriend que esa mujer staring into the fishtank a esas raras criaturas está chiflada. Seguro que le está diciendo something like that.

¿Pequeñito reptil? No. Minúhculo anfibio. About 10 inches long. Hay dos. Pale yellow (son albinos, luego me contará el hipster German hotel owner), entre banana slug y baguette. Y oh, cómo te encuentro aquí, at last, chiquititos, bichitos raros, with your 
pale, smooth, mottled skin, tus teensy froggy forelegs y cuatro deditos like a doll's starfish. Los bracitos extendidos, posed like a miniature Gila monster, like a South African likkewaan through the looking glass, mirando fijamente hacia arriba, hacia ninguna parte. Tienes tres star-prong branquias above each would-be oreja. De repente, rítmica, involuntariamente se caen patrás, flat to your triangular head. Se agitan las cilias delicadas, ínfimas, rosadas como mucosas, like the inside of a chirimoya o una guayaba. Your tiny, gold-disk eyes de centro rosado siguen mirando fijamente.

En eso, Julio, about their eyes, tuviste razón. Pero no sé (ay, argentinihmo) ... no sé si en todo lo demás. Aquí, ahora, here in Buenos Aires, vengo a descubrir que eso que escribiste eras todo vos. (Well, what/who the hell else did you expect it to be, nena?) Bueno OK, sí, admito que hay una fuerte pulsión de espiritualidad in that gaze, en esa praying mantis, mini-legavaan pose, en este absolute stillness que mira, looks right into me, through me, past me.

Suddenly, out of the acuario-shadows otro de Uds. se lanza en movimiento. (De esta modalidad, Julio, nunca escribiste.) Rapidísimo te desplazás, meneando la colita de polliwog like a hula dancer. Vos, Black Beauty, I've never heard of your kind. (Pero ¿de qué color se supone que deben ser? No me acuerdo) Me tinca que sos varón. Además, hombre atrapado. Contenido. Ahí dentro. Como boxeador. Like an outclassed middle-weight against the ropes. $\mathrm{O}$ un toro acorralado entre picador y banderillero. Ay, Black Beauty. Acometés, branquias flattened, teensy tiburón. Tu flat, wide Aztec boca slightly open, tus negros, pencil-lead ojos straight ahead. I bend and crane my head. Mi café irlandés se enfría en la otra mesa. Pero I can’t get inside esos ojitos negros. Ay, mini-dinosaur, te lanzás. Tropezás contra el cristal. Tus delicados dedos rozan, no penetran.

Tus blondas girlfriends estólidas, fofas y vos tanto embiste tanta ansia, insatisfacción en tu pequeño cuerpo. Pero no sos mutable. Ninguna metamorfosis posible. Tanto rozar y chocar pero no lográs salir de ahí ni sho entrar. En vos.

Julio, you were wrong, carnal. O este no es el que vos viste, the one you switched places with en aquel Jardín des Plantes de París. 
A vos, Black Beauty, te bautizo mi axolotl porteño. Sos como yo. Bicho oximorónico, fronterizo, uncomfy, intersticial. En constante movimiento. Los de Julio apenitas se movían, sluggishly rozándose, politely ASUMIDOS en esa su essential inmobilidad parisina. Pero vos no. Can’t keep track of you. Mercurio. Tu pasión es palpable.

Y ahora heme aquí. En el Boquitas Pintadas Pop Hotel en la calle Estados Unidos en el barrio de Monserrat, Buenos Aires. Y estas rubias, calladas criaturas, mos def femeninas. Raised up on their fragile, transparent forearms. Parece que rezan. Meditan en el más ashá ...

Axolota: versión \#2

Or: are you the girl, Black Beauty? ¿me habré quedado identificándome inconsciente, pendejamente con el (sha superado, OB-vio, y tan politically incorrect) Lector $\mathrm{MACHO}$ ?

The horror, the horror... Ay, why did I do this? Pero tan poco inspiring la otra alternativa, ¿no? La insípida, 'irracional,' predictably FEMININE, dreaded Lector HEMBRA. Just the word makes me tremble with rage, con toda esa y su fuerza atávica, biológica. How could you, Julio?

La poeta Andrea Gutiérrez insiste en que el negro-mi negro-es hembra. Y no sólo eso: she says she's big like that — henchida $-\mathrm{y}$ activa porque she's pregnant y busca escaparse de los confines del aquarium para parir. The blond ones, en cambio, según esta versión muy a lo Monique Wittig, muy amazónica, serían unos concubinos súbditos. Y por eso tan teensy, so docile. Me intriga esta teoría. Pero confieso que I'm shaken. No sé si me convence del todo...

¿Seré una convencional? Una boludehcamente happily-ever-after kinda girl, after all?

La dueña alemana concuerda contundentemente con mi versión, pero she freely admits que they've never had babies. Y finalmente, after much quizzing, confiesa que directamente no se sabe si son machos, hembras, hermafroditas o in-between. Y el dueño alemán, her hipster hubby, tells us emphatically que lo único que se sabe es que no se sabe. 
Here I am, gazing embelesada a estos prehistoric Mexican axolotl, pero no en un acuario en París: so much your city, Julio, y la tuya, Alejandra. And, for a brief time, en ese icy, cold-water flat winter de 1983, my city too, con mi prima Lee, remember James? Ahora estoy en un hotel bar en Buenos Aires, city of my sueños. This city still (y)our city, aquí en el Sur, oh, a pesar de, no obstante - o quizás por-vuestra, nuestra indiscutible extranjería.

Sin explicación posible (ni necesaria), then. Sho. Buenos Aires. Vos. Y ellos, los axolotl. Buenos Aires: the force of inevitability pero pronto, ay, way too soon, la partida. Perhaps por eso esto ahora, entonces. The dense, compact perfection of this rush of experience and memory. Toda esta desconcertante, bewitching, non-coincidental simultaneidad.

Ambiguas criaturas. Bichos raros. Ambas readings, entonces. Both/and. Y más. Siempre más. 